\title{
Shared Human Leukocyte Antigen (HLA) Coverage in dementia and Parkinson's disease
}

\author{
Lisa M. James ${ }^{1,2,3}$, Apostolos P. Georgopoulos $1,2,3,4 *$ \\ 'Brain Sciences Center, Department of Veterans Affairs Health Care System, Minneapolis, MN, 55417, USA \\ ${ }^{2}$ Department of Neuroscience, University of Minnesota Medical School, Minneapolis, MN 55455, USA \\ ${ }^{3}$ Department of Psychiatry, University of Minnesota Medical School, Minneapolis, MN 55455, USA \\ ${ }^{4}$ Department of Neurology, University of Minnesota Medical School, Minneapolis, MN 55455, USA
}

\section{Article Info}

\section{Article Notes}

Received: August 12, 2020

Accepted: September 22, 2020

\section{*Correspondence:}

Dr. Apostolos P. Georgopoulos, Brain Sciences Center (11B), Minneapolis VAHCS, One Veterans Drive, Minneapolis, MN 55417, USA; Email: omega@umn.edu.

(c) 2020 Georgopoulos AP. This article is distributed under the terms of the Creative Commons Attribution 4.0 International License

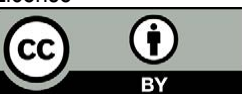

\section{Keywords}

Dementia

Parkinson's Disease

Human Leukocyte Antigen

Immunity

Genetics

\section{ABSTRACT}

Dementia and Parkinson's disease are the two most common age-related neurodegenerative conditions. Recent studies have identified Human Leukocyte Antigen (HLA) Class II DRB1 alleles that are protective or neutral with respect to dementia. Here we extend those findings to evaluate the association of the population frequency of HLA DRB1 alleles with the prevalence of dementia and Parkinson's disease in14 Continental Western European countries. Nine HLA DRB1 alleles were identified including four that are protective against dementia (DRB1*01:01, DRB1*04:01, DRB1*13:02, DRB1*15:01), three that are neutral (DRB1*03:01, DRB1*07:01, DRB1*08:01), and two susceptibility alleles (DRB1*11:01, DRB1*04:05). Results demonstrated that the population prevalence's of dementia and Parkinson's disease are highly correlated and that the association between the nine DRB1 alleles above and the population prevalence of dementia is highly overlapping with that of Parkinson's disease. These findings suggest a common HLA Class II DRB1 profile. Given the diverse role of HLA Class II alleles in protection from foreign antigens, autoimmunity, and, possibly, neuroprotection, the shared HLA profile between dementia and Parkinson's disease indicates that common immunogenetic mechanisms underlie the pathogenesis and manifestation of these diseases.

\section{Introduction}

Dementia and Parkinson's disease are the most common age-related neurodegenerative conditions, together affecting an estimated 50 million people worldwide according to recent epidemiological studies ${ }^{1,2}$. Dementia refers to conditions reflecting a decline in cognitive, behavioral, motor, and/or social functioning; Alzheimer's disease is the most common type, accounting for up to $80 \%$ of dementia cases ${ }^{3}$. AD is characterized primarily by impairments in memory and executive functioning although effects on mood, personality, and motor symptoms are also common. Parkinson's disease is characterized primarily by motor symptoms including bradykinesia, rigidity, and tremor, yet non-motor symptoms including cognitive and mood disruptions are common, particularly as the disease progresses ${ }^{4}$. A large percentage of patients with Parkinson's Disease eventually develop dementia ${ }^{5}$. In light of the overlap in symptoms and the substantial and increasing global burden of these conditions ${ }^{1,2}$, research aimed at identifying putative risk and protective factors are of paramount importance to mitigate the significant impacts of these condition on global health.

Although the etiology of dementia and Parkinson's disease is 
unknown, various genetic and environmental risk and protective factors have been identified. For dementia, risk factors include possession of apolipoprotein E4 allele, health conditions such as cardiovascular disease and Type II diabetes, modifiable lifestyle factors such as poor diet and sedentary lifestyle, and exposure to infectious agents ${ }^{6,7}$. For Parkinson's disease, variations in genes associated with alpha-synuclein and other proteins have been implicated ${ }^{8}$, and environmental risk factors include exposure to pollutants, pesticides, and infectious agents, dairy consumption, and $\beta 2$ - adrenoreceptor agonists $(2,9,10,11)$. With regard to protection, adherence to plant-based diet as well as physical, social, and intellectual activities have been shown to protect against dementia ${ }^{6}$; environmental factors that protect against Parkinson's disease include smoking, caffeine and tea intake, physical activity, vitamin $\mathrm{E}$ intake, gout, non-steroidal anti-inflammatory drugs and $\beta 2$ - adrenoreceptor agonists ${ }^{10}$. In terms of genetic protection, studies have demonstrated that apolipoprotein E2 protects against dementia ${ }^{12,13}$ and may protect against Parkinson's disease-related dementia ${ }^{14,15}$. Moving beyond apolipoprotein E, recent studies have also demonstrated robust genetic protection against dementia conferred by specific Class II human leukocyte antigen (HLA) alleles ${ }^{16,17}$. The extent to which those HLA alleles protect against Parkinson's disease is unknown.

HLA genes code for cell-surface glycoproteins that are instrumental in immune response to foreign antigens such as viruses and bacteria. Class I HLA proteins present intracellular foreign antigen peptides to cytotoxic $\mathrm{T}$ cells to signal cell destruction. Class II HLA proteins present peptides to $\mathrm{CD} 4$ receptors to promote antibody production and elimination of foreign antigens. However, HLA genes, which are the most highly polymorphic in the human genome, differ in their ability to bind with foreign antigens and mount an immune response. In the absence of sufficient binding affinity and/or immunogenicity, antigenic peptides may persist. We have speculated that persistent antigens lead to neuronal damage and inflammation, and may contribute to dementia and other neuroimmune conditions $^{18-20}$.

Using a publicly available repository ${ }^{21}$ containing HLA data pooled from histocompatibility laboratories and published studies, coupled with dementia prevalence obtained from the Global Burden of Disease studies ${ }^{1}$ we have examined associations between HLA and dementia prevalence in 14 countries in Continental Western Europe where dementia prevalence is among the highest in the world. We demonstrated that three specific Class II HLA DRB1 alleles - DRB1*01:01, DRB1*13:02, and DRB1*15:01 - are highly protective against dementia, even after accounting for apolipoprotein E $4{ }^{17}$. Moreover, the combined population frequency of the three alleles accounted for $67 \%$ of the variance in dementia prevalence and $48 \%$ of the change in dementia prevalence in those countries over the last 25 years. Given the role of Class II HLA in antibody production and host protection against re-exposure to foreign antigens, the finding that dementia prevalence is lower in countries with higher frequency of these alleles suggests that lower dementia rates may be partially attributable to protection against pathogens that bind with these alleles. In fact, a subsequent in silico study revealed that these three dementia alleles were found to bind with significantly higher affinity to herpes virus antigens than were three HLA alleles that are dementia-neutral - DRB1*03:01, DRB1*07:01, DRB1*08:0122. In the present study, we extend those findings to investigate the association of the population frequency ${ }^{21}$ of HLA -DRB1 dementiaprotective, dementia-neutral alleles to Parkinson's disease population prevalence ${ }^{2}$.

\section{Methods}

CWE countries. As in our previous studies ${ }^{16,17}$ we focused on the following 14 CWE countries: Austria, Belgium, Denmark, Finland, France, Germany, Greece, Italy, Netherlands, Portugal, Norway, Spain, Sweden, and Switzerland.

Prevalence of dementia and Parkinson's disease. The Global Burden of Disease study is the most comprehensive epidemiological study of worldwide morbidity and mortality associated with several conditions including dementia $^{1}$ and Parkinson's disease ${ }^{2}$. The total number of people with dementia or Parkinson's disease in each of the $14 \mathrm{CWE}$ countries was retained for analyses in the present study. The Population Reference Bureau ${ }^{23}$ provides vital demographic data for over 200 countries based on several sources including official national data or analyses conducted by national, regional, or worldwide (e.g. United Nations Population Division) offices. The prevalences of dementia and Parkinson's disease were computed by dividing the number of people with dementia ${ }^{1}$ or Parkinson's disease ${ }^{2}$ in 2016 in each country by the total population of the country in $2016^{23}$ and expressed as a percentage. These data are shown in Table 1 . We have previously shown that life expectancy for these countries are virtually identical ${ }^{3}$ (Table 1 ); therefore, life expectancy was not included in the current analyses.

HLA alleles. In our previous studies we identified three HLA Class II alleles protective for dementia ${ }^{17}$ (DRB1*01:01, DRB1*13:02, DRB1*15:01) and three alleles that are neutral for dementia ${ }^{21}$ (DRB1*03:01, DRB1*07:01, DRB1*08:01); in addition, we now identified an allele protective for dementia (DRB1*04:01; Allele Frequency Net Database, http://www.allelefrequencies.net ${ }^{21}$ ). To obtain allele frequencies, the Allele Frequency Net Database was 
Table 1. Dementia prevalence for Countries in Continental Western Europe. Overall Dementia Prevalence $=(\mathrm{N}$ Disease $\times 100) /(\mathrm{N}$ total population)

\begin{tabular}{|c|c|c|c|c|c|c|}
\hline Country & N Dementia $^{\mathbf{a}}$ & $\begin{array}{c}\mathbf{N} \\
\text { Parkinson's Disease }^{\mathbf{b}}\end{array}$ & $\begin{array}{c}\mathbf{N} \\
\text { total population }^{\mathbf{c}}\end{array}$ & $\begin{array}{c}\text { Dementia } \\
\text { Prevalence (\%) }\end{array}$ & $\begin{array}{c}\text { Parkinson's Disease } \\
\text { Prevalence (\%) }\end{array}$ & $\begin{array}{c}\text { Average life expectancy } \\
\text { (\%) }\end{array}$ \\
\hline Austria & 126914 & 15891 & 8800000 & 1.442 & 0.1806 & 81.5 \\
\hline Belgium & 181350 & 20862 & 11300000 & 1.605 & 0.1846 & 81.5 \\
\hline Denmark & 55336 & 9068 & 5700000 & 0.971 & 0.1591 & 80.5 \\
\hline Finland & 83950 & 10258 & 5500000 & 1.526 & 0.1865 & 81.5 \\
\hline France & 877760 & 120455 & 64600000 & 1.359 & 0.1865 & 82.0 \\
\hline Germany & 1201668 & 162246 & 82600000 & 1.455 & 0.1964 & 80.5 \\
\hline Greece & 192563 & 22837 & 10800000 & 1.783 & 0.2115 & 81.0 \\
\hline Italy & 1370308 & 144606 & 60600000 & 2.261 & 0.2386 & \\
\hline Netherlands & 192425 & 33297 & 17000000 & 1.132 & 0.1959 & 82.5 \\
\hline Norway & 67207 & 7517 & 5200000 & 1.292 & 0.1446 & 81.5 \\
\hline Portugal & 166660 & 18496 & 10300000 & 1.618 & 0.1796 & 82.0 \\
\hline Spain & 830915 & 92971 & 43300000 & 1.919 & 0.2147 & 80.0 \\
\hline Sweden & 142735 & 19776 & 9900000 & 1.442 & 0.1998 & 82.5 \\
\hline Switzerland & 115476 & 14979 & 8400000 & 1.375 & 0.1783 & 82.0 \\
\hline
\end{tabular}

${ }^{a}$ Data obtained from ref ${ }^{1}$.

${ }^{\mathrm{b}}$ Data obtained from ref².

'Data obtained from ref ${ }^{\text {pop'n bureau }}$

Table 2. Frequencies of 9 HLA Class II alleles for countries in Continental Western Europe used in this study. ${ }^{a}$ /A, not available.

\begin{tabular}{|c|c|c|c|c|c|c|c|c|c|}
\hline Country & DRB1*01:01 & DRB1*03:01 & DRB1*04:01 & DRB1*04:05 & DRB1*07:01 & DRB1*08:01 & DRB1*11:01 & DRB1*13:02 & DRB1*15:01 \\
\hline Austria & 0.102 & .118 & 0.068 & N/A & .134 & .015 & .090 & 0.062 & 0.115 \\
\hline Belgium & 0.086 & .157 & 0.061 & .015 & .111 & .030 & .071 & 0.035 & 0.123 \\
\hline Denmark & 0.130 & .102 & 0.176 & .000 & N/A & .028 & .009 & 0.056 & 0.176 \\
\hline Finland & 0.107 & .079 & 0.119 & $\mathrm{~N} / \mathrm{A}$ & .035 & .078 & .023 & 0.025 & 0.104 \\
\hline France & 0.082 & .120 & 0.101 & .013 & .114 & .024 & .076 & 0.047 & 0.109 \\
\hline Germany & 0.092 & .103 & 0.079 & .011 & .114 & .027 & .072 & 0.043 & 0.144 \\
\hline Greece & 0.060 & .085 & 0.011 & .013 & .076 & .011 & .101 & 0.033 & 0.045 \\
\hline Italy & 0.060 & .152 & 0.022 & .039 & .103 & .022 & .135 & 0.038 & 0.055 \\
\hline Norway & 0.080 & .140 & 0.140 & .000 & .120 & .040 & .040 & 0.050 & 0.170 \\
\hline Portugal & 0.047 & .114 & 0.039 & .024 & .158 & .038 & .057 & 0.036 & 0.069 \\
\hline Spain & 0.057 & .109 & 0.030 & .018 & .192 & .024 & .058 & 0.033 & 0.140 \\
\hline Sweden & 0.085 & .078 & 0.082 & .004 & .031 & .131 & .083 & 0.038 & 0.111 \\
\hline Switzerland & 0.078 & N/A & N/A & N/A & $\mathrm{N} / \mathrm{A}$ & N/A & .076 & 0.053 & 0.109 \\
\hline
\end{tabular}

a. Obtained from allelefrequencies.net October 19, 2019.

queried to provide the 4-digit HLA allele frequency for each country with no further restrictions by region, ethnicity, source of study, or other qualifiers. For countries in which were available for more than one population we obtained the average frequency. The frequencies of these seven alleles for each country are given in Table 2.

Statistical analyses. Regression and correlation analyses were used to evaluate the association between the population frequency of each the seven HLA alleles above (Table 2), alone and combined, and the prevalence of dementia and Parkinson's disease in Continental Western Europe. Previous analyses ${ }^{16,17}$ had documented an exponential relation between dementia prevalence and allele frequency; hence, the prevalences of dementia and Parkinson's disease were natural-log transformed to analyze their relation to allele frequency using linear regression. Pairwise Pearson correlation coefficients were compared by calculating the normal deviate $z$-statistic ${ }^{24}$.
Regression coefficients were compared, as needed, using the following formula ${ }^{25}$ :

$$
z=\frac{b_{1}-b_{2}}{\sqrt{S E b_{1}{ }^{2}+S E b_{2}^{2}}}
$$

where $z$ is the normal deviate, $b_{1}$ and $b_{2}$ denote regression coefficients to be compared, and $S E$ are their standard errors. Finally, correlation coefficients $r$ were Fisher z-transformed ${ }^{24} r^{\prime}$ to normalize their distribution:

$$
r^{\prime}=\operatorname{atanh}(r)
$$

All statistical analyses were conducted using the IBMSPSS statistical package (version 23).

\section{Results}

Association of dementia and Parkinson's disease prevalences

The prevalences of dementia and Parkinson's disease 


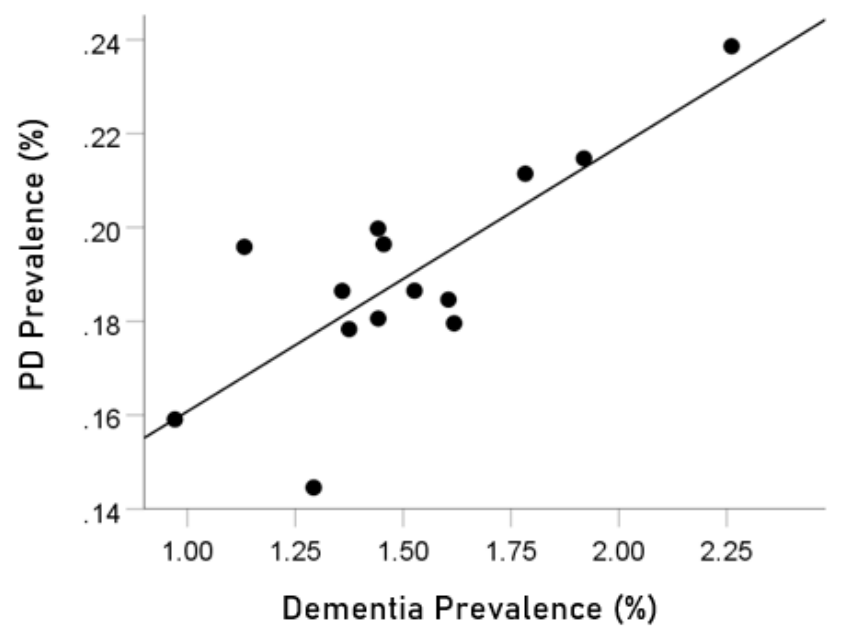

Figure 1. Dementia prevalence in 14 CWE countries is plotted against the prevalence of Parkinson's disease. See text for details.

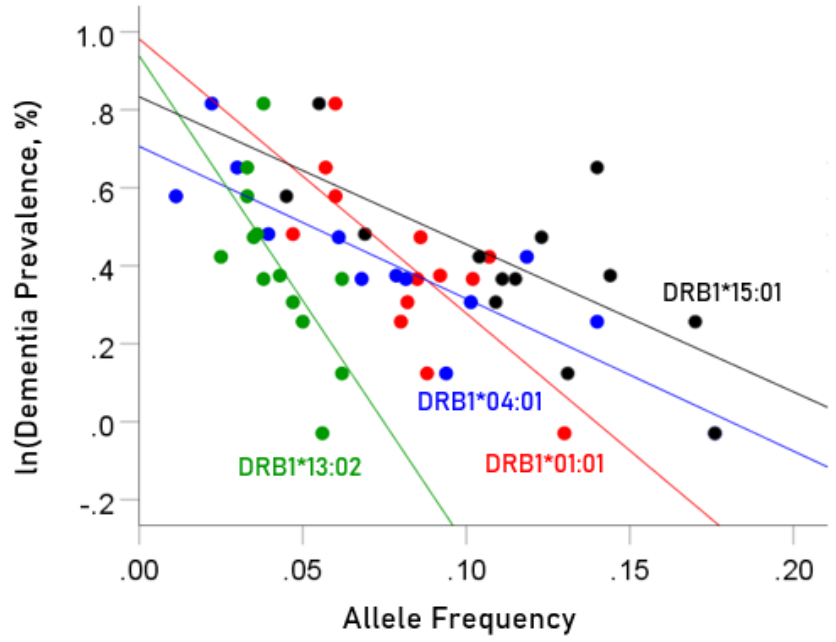

Figure 2. Illustration of the different effects of each protective allele on the natural log of dementia prevalence as evidenced by the varying steepness of the slopes.

Table 3. Correlation statistics between disease prevalences and allele frequencies analyzed. P-values are in parentheses. Blue, protective alleles; black, neutral; red, predisposing.

\begin{tabular}{|c|c|c|c|c|c|c|}
\hline & \multirow{3}{*}{ Alleles } & \multicolumn{2}{|c|}{ Correlation Coefficients } & \multicolumn{2}{|c|}{ Comparison of Correlations } & \multirow[b]{3}{*}{$\mathbf{N}$} \\
\hline & & \multirow{2}{*}{$\begin{array}{l}\text { In(Dementia prevalence) } \\
\text { HLA Profile for Dementia }\end{array}$} & \multirow{2}{*}{$\begin{array}{c}\text { In(Parkinson's disease prevalence) } \\
\text { HLA Profile for Parkinson's }\end{array}$} & & & \\
\hline & & & & Test statistic $z$ & $P$ value & \\
\hline 1 & DRB1*01:01 & $-0.727(P=0.003)$ & $-0.480(0.082)$ & 0.937 & 0.174 & 14 \\
\hline 2 & DRB1*04:01 & $-0.857(0.00018)$ & $-0.778(0.002)$ & 0.570 & 0.284 & 13 \\
\hline 3 & DRB1*13:02 & $-0.674(0.008)$ & $-0.428(0.127)$ & 0.846 & 0.199 & 14 \\
\hline 4 & DRB1*15:01 & $-0.686(0.007)$ & $-0.640(0.014)$ & 0.193 & 0.424 & 14 \\
\hline 5 & DRB1*03:01 & $0.102(0.739)$ & $-0.057(0.854)$ & 0.356 & 0.361 & 13 \\
\hline 6 & DRB1*07:01 & $0.212(0.509)$ & $-0.073(0.821)$ & 0.612 & 0.270 & 12 \\
\hline 7 & DRB1*08:01 & $-0.086(0.780)$ & $-0.046(0.882)$ & 0.090 & 0.464 & 13 \\
\hline 8 & DRB1*11:01 & $0.670(0.009)$ & $0.705(0.005)$ & 0.156 & 0.438 & 14 \\
\hline 9 & DRB1*04:05 & $\begin{array}{c}0.854 \\
(0.001)\end{array}$ & $\begin{array}{l}0.676 \\
(0.022)\end{array}$ & 0.898 & 0.185 & 11 \\
\hline
\end{tabular}

were highly significantly correlated (Figure $1 ; \mathrm{r}=0.787, \mathrm{P}$ $=0.000843, \mathrm{~N}=14$ ).

\section{Protective HLA alleles}

Association between dementia and Parkinson's disease prevalences and the frequencies of four protective HLA alleles

The values of log-transformed dementia and Parkinson's disease prevalences are plotted against the frequencies of the four protective (negatively associated) alleles in Figure. 2 and 3, respectively. The corresponding correlation coefficients are given in Table 3 . There were no statistically different differences between any of the dementia- and Parkinson's disease correlations (Table 3).

Association of dementia and Parkinson's disease prevalences with the combined frequencies of the four protective alleles

The log-transformed prevalence of dementia is plotted

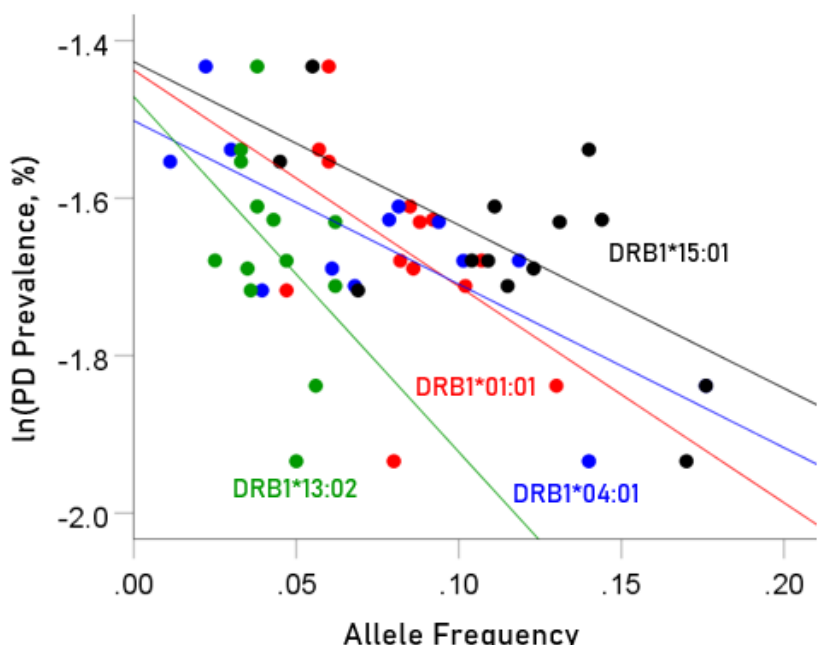

Figure 3. Illustration of the different effects of each protective allele on the natural log of Parkinson's disease prevalence as evidenced by the varying steepness of the slopes. 
against the sum of the frequencies of four protective alleles in Figure 4:

$f_{[4 \text { protective alleles }]}=f_{D R B 1 * 01: 01}+f_{D R B 1 * 04: 01}+f_{D R B 1 * 13: 02}+f_{D R B 1 * 15: 01}$

The regression equation was as follows

$\ln ($ dementia prevalence, $\%)=0.963-1.766( \pm 0.301)$ $f_{[4 \text { protective alleles }]}+$ error

$\mathrm{r}^{2}=0.758, \mathrm{P}=0.001$; value in parenthesis is the SE of the regression coefficient.

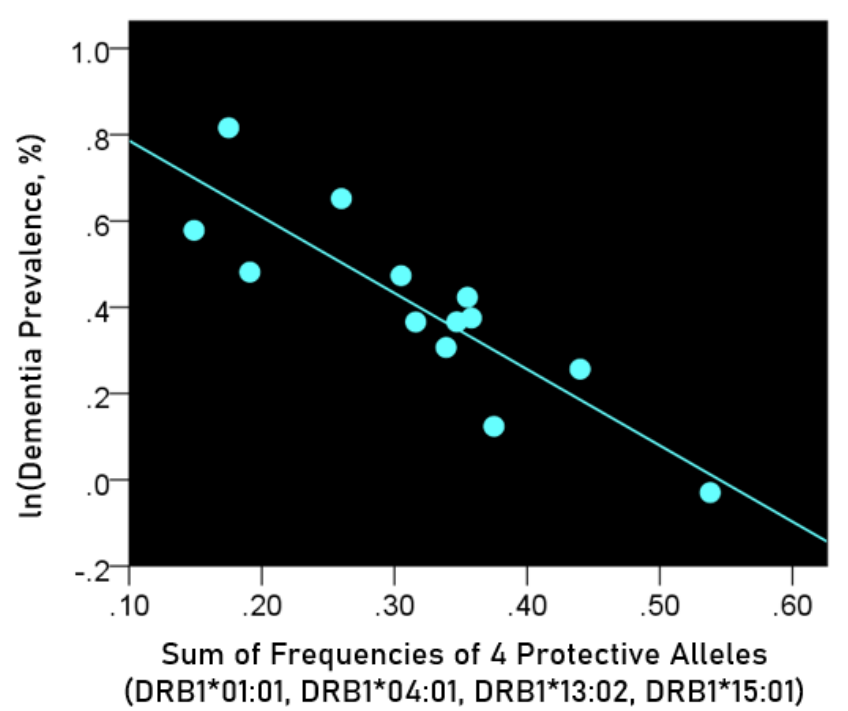

Figure 4. The natural log of dementia prevalence is plotted against the sum of the frequencies of the four protective alleles (DRB1*01:01, DRB1*04:01, DRB1*13:02, DRB1*15:01). See text for details.

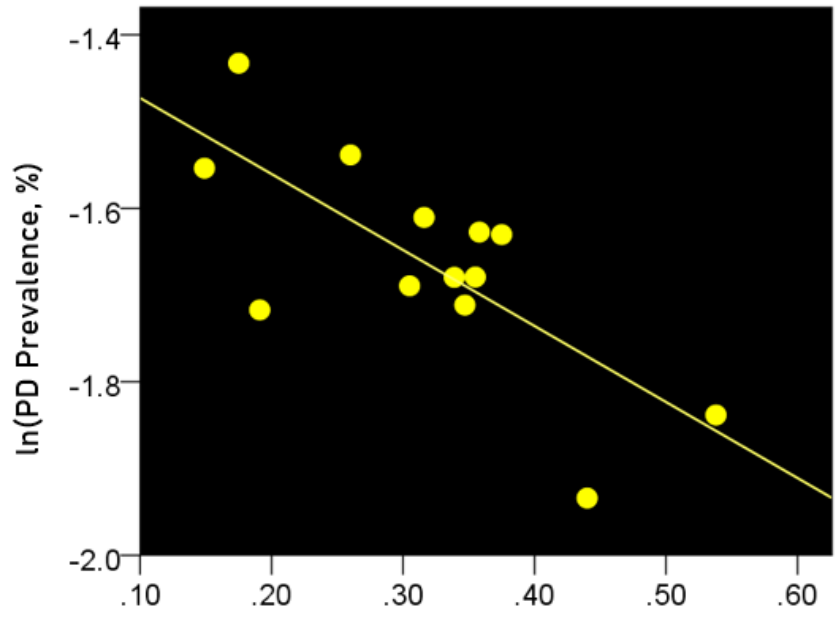

Sum of Frequencies of 4 Protective Alleles (DRB1*01:01, DRB1*04:01, DRB1¹3:02, DRB1*15:01)

Figure 5. The natural log of Parkinson's disease prevalence is plotted against the sum of the frequencies of the four protective alleles (DRB1*01:01, DRB1*04:01, DRB1*13:02, DRB1*15:01). See text for details.
The log-transformed prevalence of Parkinson's disease (PD) is plotted against the sum of the frequencies four protective alleles in Figure 5. The regression equation was as follows.

$\ln (\mathrm{PD}$ prevalence, \%) $=-1.385-0.876( \pm 0.241)$

$f_{[4 \text { protective alleles }]}+$ error

$\mathrm{r}^{2}=0.546, \mathrm{P}=0.004$; value in parenthesis is the SE of the regression coefficient.

The $f_{\text {[4 protectivealleles }]}$ regression coefficient for dementia ( -1.766 , equation 3 ) was 2 x more negative (i.e. twice as protective) for dementia than that for Parkinson's disease $(-0.876$, equation 4$)$, a highly significant difference $(z=5.36, P<0.001$, test of equation 1$)$.

\section{Neutral alleles}

Association between dementia and Parkinson's disease prevalences and the frequencies of three neutral HLA alleles

The values of log-transformed dementia and Parkinson's disease prevalences are plotted against the frequencies of the three neutral (not significantly associated) alleles in Figure. 6 and 7, respectively. The corresponding correlation coefficients are given in Table 3 . There were no statistically different differences between any of the dementia- and Parkinson's disease correlations (Table 3).

Association of dementia and Parkinson's disease prevalences with the combined frequencies of the three neutral alleles

The log-transformed prevalence of dementia is plotted against the sum of the frequencies of the three neutral alleles in Figure 8:

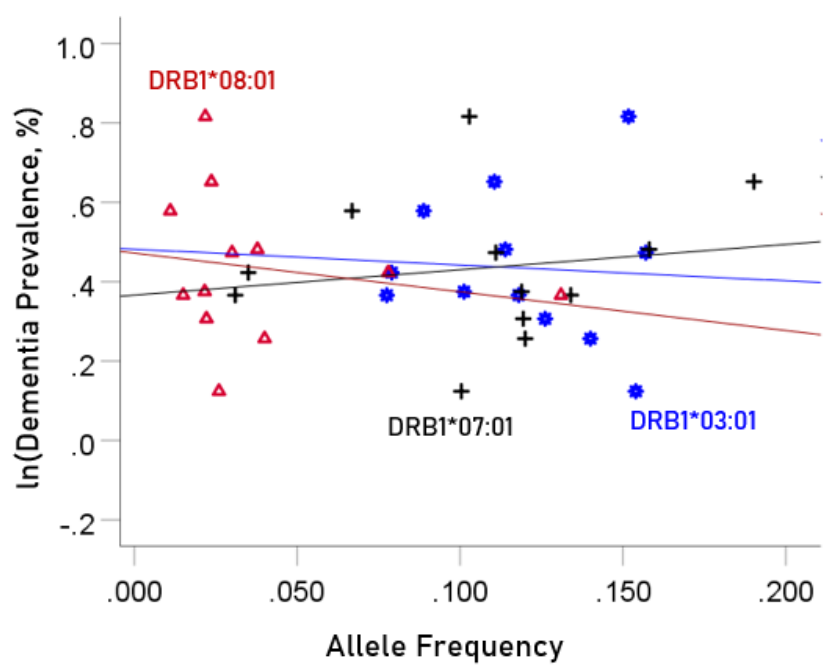

Figure 6. The natural log of dementia prevalence is plotted against the frequency of the three neutral alleles; there is no significant association. See text for details. 


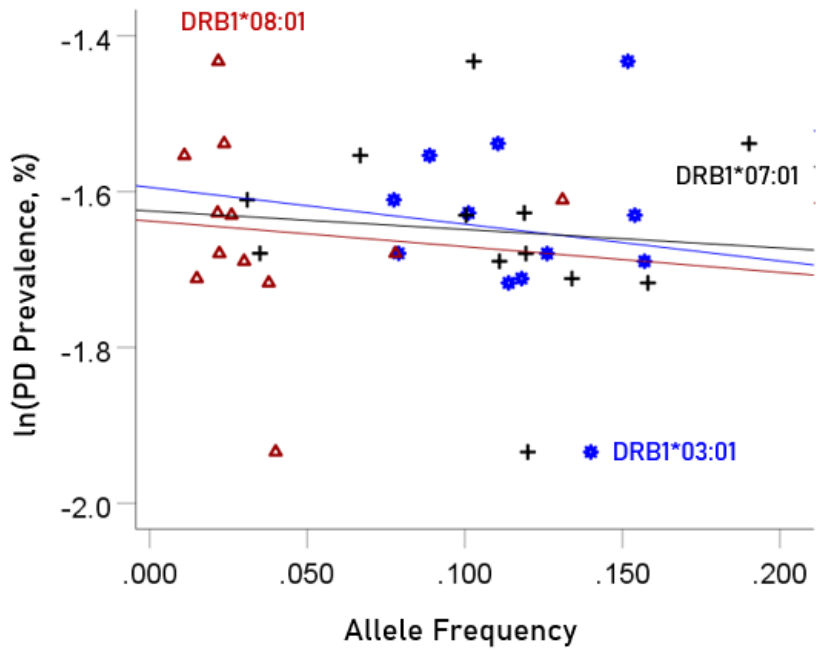

Figure 7. The natural log of Parkinson's disease prevalence is plotted against the frequency of the three neutral alleles; there is no significant association. See text for details.

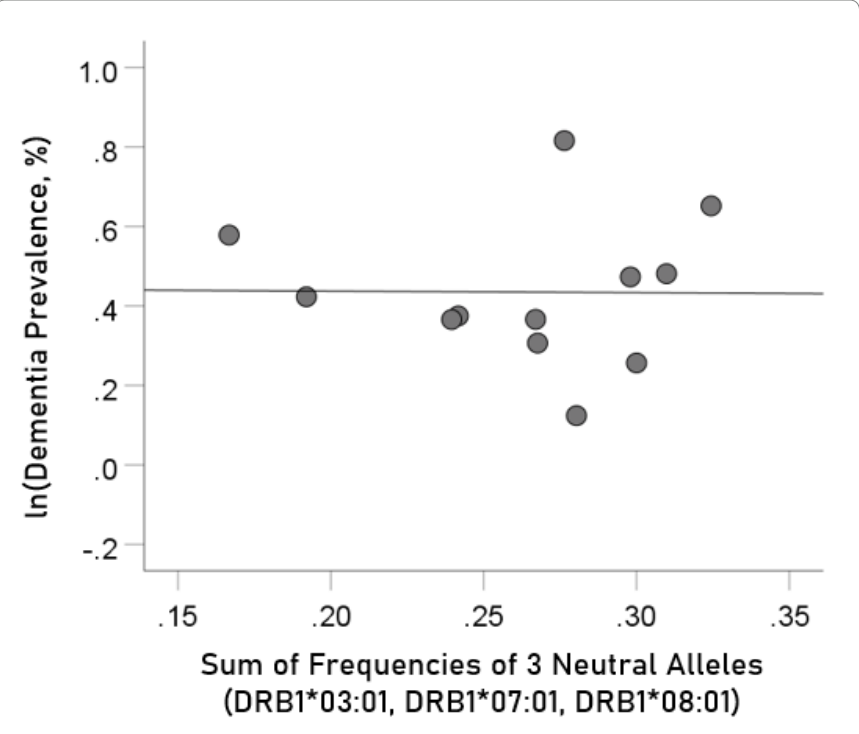

Figure 8. The natural log of dementia prevalence is plotted against the sum of the frequencies of the three neutral (DRB1*03:01, DRB1*07:01, DRB1*08:01); there is no significant association. See text for details.

$$
f_{[3 \text { neutralalleles }]}=f_{D R B 1 * 03: 01}+f_{D R B 1 * 07: 01}+f_{D R B 1 * 08: 01}
$$

The regression equation was as follows.

$\ln ($ dementia prevalence, $\%)=0.446-0.41( \pm 1.239)$ $f_{[3 \text { neutralalleles }]+\text { error }}$

$\mathrm{r}^{2}=0.0001, \mathrm{P}=0.974 ;$ value in parenthesis is the SE of the regression coefficient. There was no statistically significant association.

The log-transformed prevalence of Parkinson's disease is plotted against the sum of the frequencies four protective

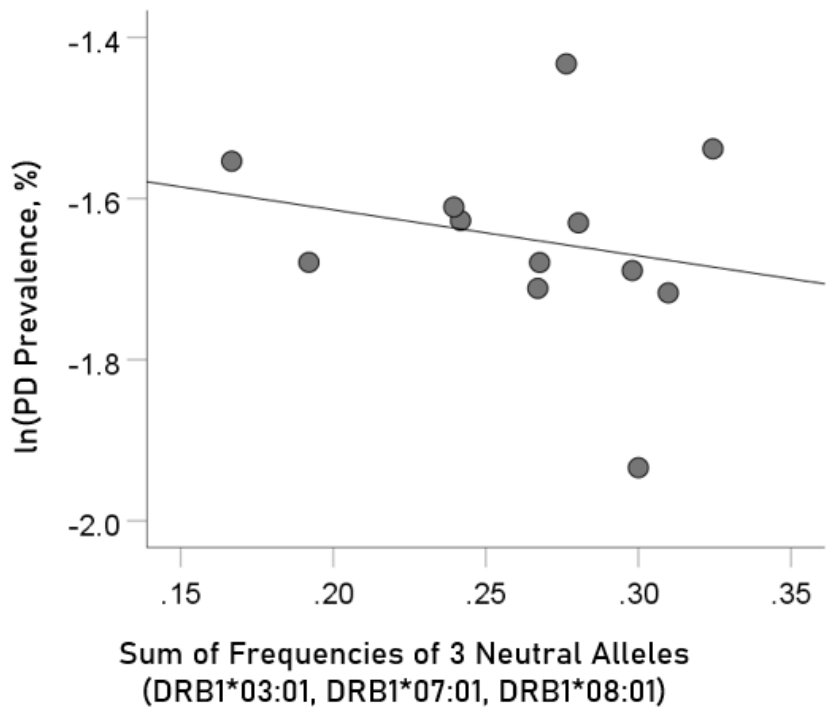

Figure 9. The natural log of Parkinson's disease prevalence is plotted against the sum of the frequencies of the three neutral (DRB1*03:01, DRB1*07:01, DRB1*08:01); there is no significant association. See text for details.

alleles in Figure 9. The regression equation was as follows.

$\ln ($ Parkinson's disease prevalence, $\%)=-1.5-0.571( \pm$ 0.799) $f_{[3 \text { neutralalleles }]+\text { error }}$

$r^{2}=0.049, \mathrm{P}=0.491$; value in parenthesis is the SE of the regression coefficient. There was no statistically significant association.

\section{Predisposing HLA alleles}

Association between dementia and Parkinson's disease prevalences and the frequencies of two predisposing HLA alleles

The values of log-transformed dementia and Parkinson's disease prevalences are plotted against the frequencies of the two predisposing alleles in Figure. 10 and 11, respectively. The corresponding correlation coefficients are given in Table 3. There were no statistically different differences between any of the dementia- and Parkinson's disease correlations (Table 3).

Association of dementia and Parkinson's disease prevalences with the combined frequencies of the three neutral alleles

The log-transformed prevalence of dementia is plotted against the sum of the frequencies of the three neutral alleles in Figure 12:

$$
f_{[2 \text { predisposing alleles }]}=f_{D R B 1 * 04: 05}+f_{D R B 1 * 11: 01}
$$

The regression equation was as follows.

$\ln ($ dementia prevalence, $\%)=-0.005+4.939( \pm 1.002)$

$f_{[2 \text { predisposing alleles }]+\text { error }}$ 


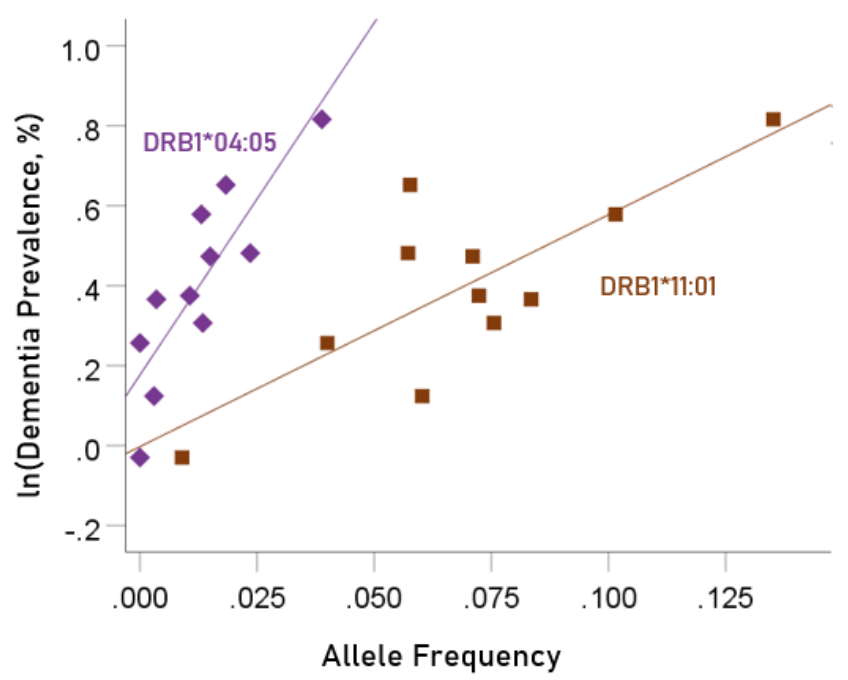

Figure 10. Illustration of the different effects of each predisposing allele on the natural log of dementia prevalence as evidenced by the varying steepness of the slopes.

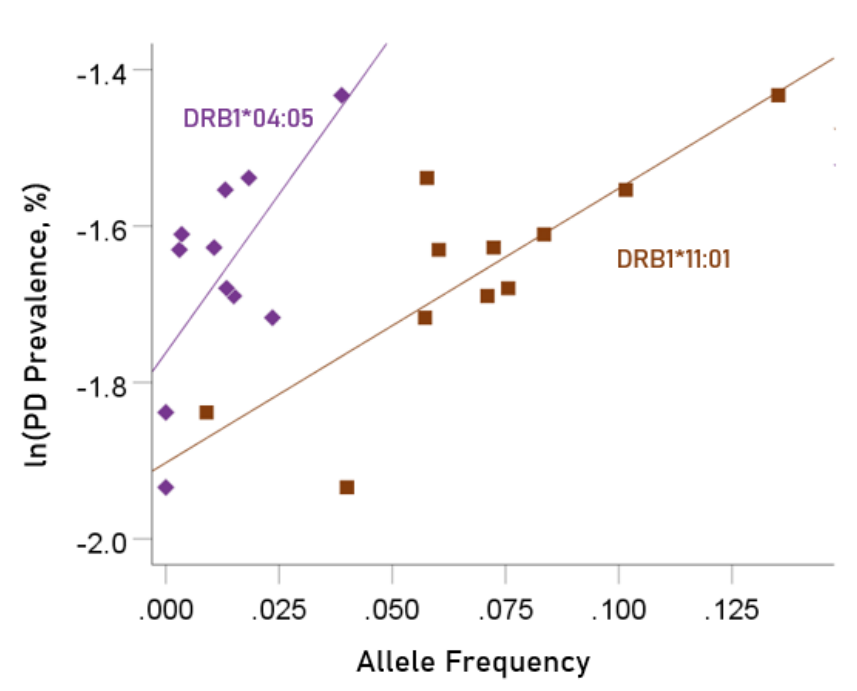

Figure 11. Illustration of the different effects of each predisposing allele on the natural log of Parkinson's disease prevalence as evidenced by the varying steepness of the slopes.

$r^{2}=0.729, P=0.001$; value in parenthesis is the SE of the regression coefficient.

The log-transformed prevalence of Parkinson's disease is plotted against the sum of the frequencies four protective alleles in Figure 13. The regression equation was as follows.

$\ln ($ Parkinson's disease prevalence, $\%)=-1.888+2.789$ $( \pm 0.626) f[3$ neutralalleles $]+$ error $(11)$

$r^{2}=0.688, P=0.002$; value in parenthesis is the SE of the regression coefficient.

\section{HLA profile of a disease}

A given disease may have varying associations with

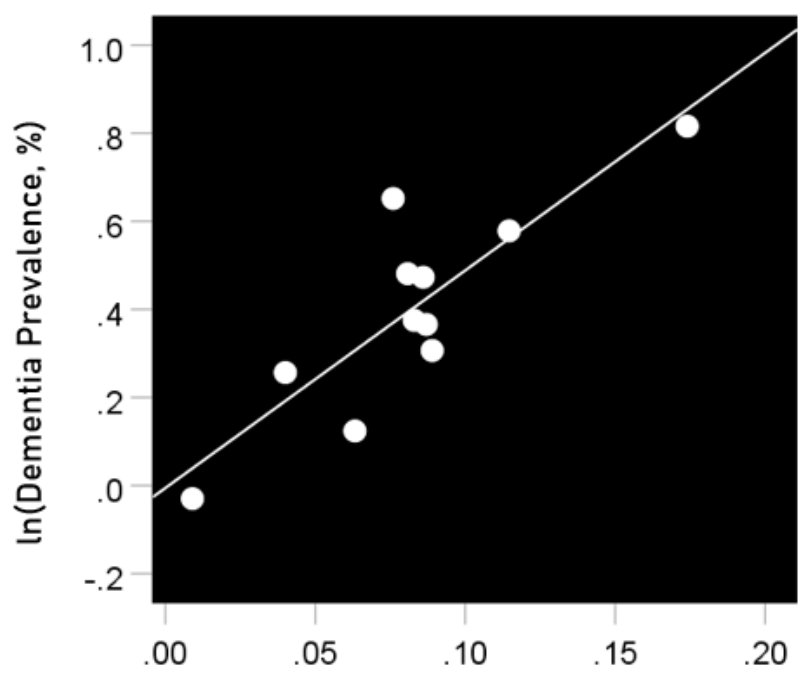

Sum of Frequencies of 2 Predisposing Alleles (DRB1*11:01, DRB*04:05)

Figure 12. The natural log of dementia prevalence is plotted against the sum of the frequencies of the two predisposing alleles (DRB1*04:05, DRB1*11:01). See text for details.

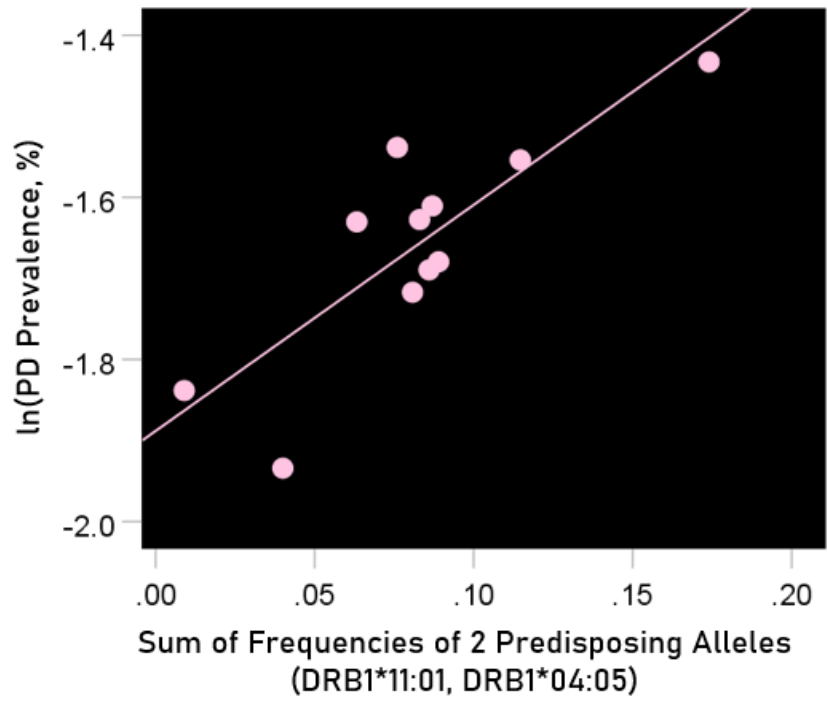

Figure 13. The natural log of Parkinson's disease prevalence is plotted against the sum of the frequencies of the two predisposing alleles (DRB1*04:05, DRB1*11:01). See text for details.

different HLA alleles. In the context of populations, this relation would be captured by the correlation between disease prevalence and allele frequency, since both of these measures typically vary across population samples (e.g. countries, ethnicities, etc.): this variation provides the requisite variety that makes disease-HLA associations possible. Let $p_{i}^{k=1, M}$ be the prevalence of disease $i$ in a sample population $K$ (out of $M$ available); let $f_{j=1, N}^{k=1, M}$ be the frequency of a HLA allele $j$ (out of $N$ available) in that population. We call the vector of $\mathrm{z}$-transformed correlations 
Table 4. HLA profiles for dementia and Parkinson's (",ease. Numbers are Fisher z-transformed correlation coefficients ${ }^{\prime}$. (See text for details.)

\begin{tabular}{|l|c|c|}
\hline & HLA Profile for Dementia & HLA Profile for Parkinson's \\
\hline DRB1*01:01 & -.0922 & -0.523 \\
\hline DRB1*03:01 & -1.286 & -1.043 \\
\hline DRB1*04:01 & -0.818 & -0.457 \\
\hline DRB1*07:01 & -0.840 & -0.758 \\
\hline DRB1*08:01 & 0.102 & -0.057 \\
\hline DRB1*13:02 & 0.215 & -0.073 \\
\hline DRB1*15:01 & -0.102 & -0.129 \\
\hline DRB1*11:01 & 0.877 & 0.811 \\
\hline DRB1*04:05 & 0.822 & 1.271 \\
\hline
\end{tabular}

$r^{\prime}$ between the prevalence of the disease $i$ in $M$ populations and the frequency of $N$ alleles in the same populations the "HLA profile of disease $i$ ". In the present study, the HLA profiles of dementia and Parkinson's disease, for the 9 HLA alleles studied, are shown in Table 4 . Specifically, $M=14$ CWE countries, $N=9$ HLA alleles.

\section{Association between HLA disease profiles}

The pairwise association between HLA disease profiles provides a quantitative estimate of the similarity of dependence of disease prevalence to an ensemble of HLA alleles. Since HLA alleles can be involved in pathogen elimination (positive aspect) and/or autoimmunity (negative aspect), determining the similarity between HLA disease profiles would provide an overall assessment of the similarity of involvement of such factors in the specific diseases in a pair. Since the HLA profile consists of z-transformed correlations, the Pearson correlation between two HLA disease profiles would give a quantitative assessment of the association, including its statistical significance and the percent of HLA profile variance explained. In this study, there was a highly positive association between the dementia and Parkinson's HLA disease profiles (Figure 14); the correlation coefficient was $0.965\left(\mathrm{P}=0.000026 ; r^{2}=0.931\right)$ and the percent of variance explained $r^{2} \times 100=93.1 \%$.

\section{Discussion}

Here we investigated the association of the population frequency of seven HLA Class II DRB1 alleles with respect to the prevalence of dementia and Parkinson's disease in 14 countries in Continental Western Europe. Several findings emerged. First, the population prevalence of dementia and Parkinson's disease were positively and highly significantly associated. Second, the average frequency of protective HLA alleles accounted for substantial variance in the population prevalence of dementia and, to a lesser extent, Parkinson's disease. Finally, the associations of disease prevalence and the HLA alleles were highly similar for dementia and Parkinson's disease. Taken together, these findings point to a common HLA profile, suggesting shared immunogenetic

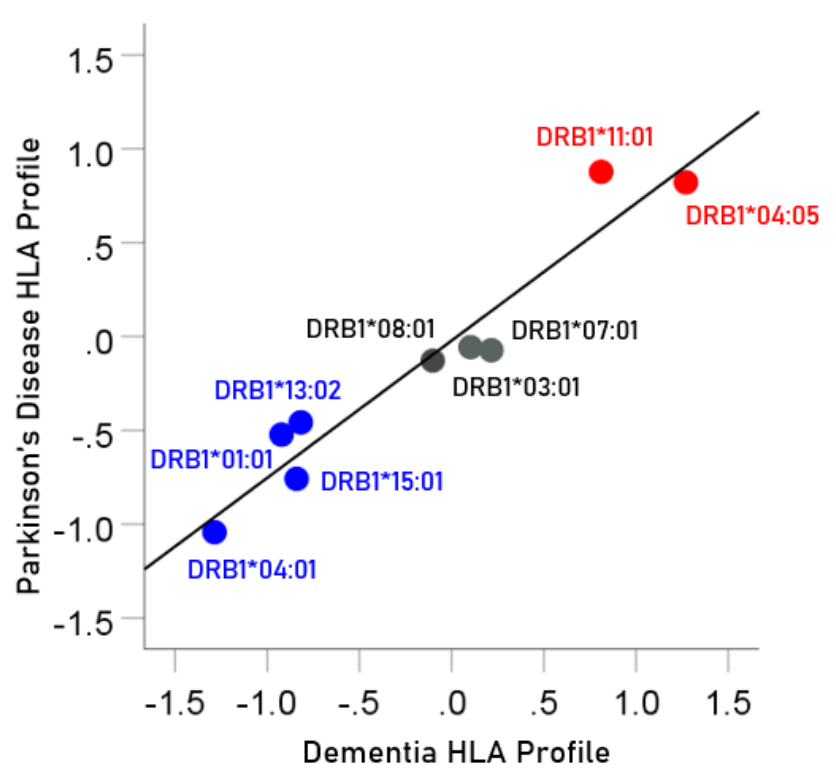

Figure 14. The HLA profile of Parkinson's disease is plotted against the HLA profile of dementia; data from Table 4. Blue, protective alleles; black, neutral alleles; red, predisposing alleles.

mechanisms (protective, neutral, and predisposing) underlying these diseases.

HLA Class II alleles, to which the DRB1 alleles investigated here belong, play a critical role in facilitating antibody production and immunological memory to foreign antigens. In light of that role, the highly protective effects of the four DRB1 alleles identified here can be interpreted as likely promoting pathogen elimination that may otherwise contribute to disease susceptibility. That is, those lacking immunogenetic protection conferred by possession of one or more of these DRB1 alleles (or other HLA alleles with similar sequences and, consequently, similar binding affinity) may be unable to sufficiently eliminate certain pathogens, leading to deleterious downstream effects that may include dementia and/or Parkinson's disease. Indeed, several viral and bacterial pathogens have been implicated in both dementia ${ }^{7}$ and Parkinson's disease ${ }^{9,11}$, many of which overlap across conditions consistent with the highly overlapping HLA profile observed here. In particular, human herpes viruses have been implicated in both conditions ${ }^{26-29}$, which is notable in light of a recent in silico study that demonstrated superior binding affinity of three of the HLA studies investigated here to human herpes viruses when compared to dementia-neutral HLA alleles ${ }^{22}$. Additional studies are currently underway to identify other antigens that bind with these protective HLA alleles and that, conversely, may be associated with dementia and/ or Parkinson's disease in the absence of HLA protection. Finally, with respect to predisposing HLA Class II alleles, such as those identified in this study, it is very likely that they are involved in autoimmunity mechanisms in both Alzheimer's and Parkinson disease, as discussed recently ${ }^{30}$. 
Although the current findings identified protective effects of four HLA DRB1 alleles, they neither preclude the possibility that other Class I or Class II HLA alleles may confer similar protection or predisposition nor that the HLA alleles shown to protect against dementia and Parkinson's disease may promote risk for other conditions. For instance, DRB1*04:01, one of the alleles shown to protect against dementia and Parkinson's disease in the current study, is associated with elevated risk of Type I diabetes ${ }^{31}$ and rheumatoid arthritis ${ }^{32}$. Similarly, DRB1*15:01 is a well-established risk factor for multiple sclerosis ${ }^{33}$, yet is protective against dementia and Parkinson's disease at the population level. Research in our laboratory is underway to examine associations of other Class I and Class II HLA alleles with dementia, Parkinson's disease, and other related conditions.

\section{Acknowledgments}

Partial funding for this study was provided by the University of Minnesota (the Anita Kunin Chair in Women's Healthy Brain Aging, the Brain and Genomics Fund, the McKnight Presidential Chair of Cognitive Neuroscience, and the American Legion Brain Sciences Chair). The sponsors had no role in the current study design, analysis or interpretation, or in the writing of this paper. The contents do not represent the views of the U.S. Department of Veterans Affairs or the United States Government. Author contributions: LMJ and APG contributed to data analysis and writing the manuscript. Conflicts of Interest: The authors declare that the research was conducted in the absence of any commercial or financial relationships that could be construed as a potential conflict of interest.

\section{References}

1. Nichols E, Szoeke CEI, Vollset SE, et al. Global, regional, and national burden of Alzheimer's disease and other dementias, 1990-2016: systematic analysis for the Global Burden of Disease Study 2016. Lancet Neurol. 2019; 18(1): 88-106.

2. Dorsey ER, Elbaz A, Nichols E, et al. Global, regional, and national burden of Parkinson's disease, 1990-2016: a systematic analysis for the Global Burden of Disease Study 2016. Lancet Neurol. 2018; 17(11): 939-53.

3. Alzheimer's Association. 2020 Alzheimer's Disease Facts and Figureres. Alzheimers Dement. 2020; 16(3): 391-460.

4. Chaudhuri KR, Healy DG, Schapira AH. Non-motor symptoms of Parkinson's disease: diagnosis and management. Lancet Neurol. 2006; 5(3): 235-45.

5. Emre M, Aarsland D, Brown R, et al. Clinical diagnostic criteria for dementia associated with Parkinson's disease. Mov Disord. 2007; 22 1689-707.

6. Livingston $G$, Sommerland A, Orgeta $V$, et al. Dementia prevention, intervention, and care. Lancet. 2017; 390(10113): 2673-2734.

7. Mawanda F, Wallace R. Can infections cause Alzheimer's disease? Epidemiol. Rev. 2013; 35: 161-80.

8. Billingsley KJ, Bandres-Ciga S, Saez-Atienzar S, et al. Genetic risk factors in Parkinson's disease. Cell Tissue Res. 2018; 373(1): 9-20.
9. Caggiu E, Arru G, Hosseini S, et al. Inflammation, infectious triggers, and Parkinson's disease. Front Neurol. 2019; 10: 122

10. Belvisi D, Pellicciari R, Fabbrini G, et al. Modifiable risk and protective factors in disease development, progression and clinical subtypes of Parkinson's disease: What do prospective studies suggest?. Neurobiol Dis. 2020; 134: 104671.

11. Wang $\mathrm{H}$, Liu X, Tan C. Bacterial, viral, and fungal infection-related risk of Parkinson's disease: Meta-analysis of cohort and case-control studies. Brain Behav. 2020; 10(3): e01549.

12. Conejero-Goldberg C, Gomar J, Bobes-Bascaran T, et al. APOE2 enhances neuroprotection against Alzheimer's disease through multiple molecular mechanisms. Mol Psychiatry. 2014; 19: 1243-1250.

13. Corder EH, Saunders AM, Risch NJ, et al, Protective effect of apolipoprotein E type 2 allele for late onset Alzheimer disease. Nat Genet. 1994; 7: 180-184.

14. Zhao N, Attrebi ON, Ren $Y$, et al. APOE4 exacerbates $\alpha$-synuclein pathology and related toxicity independent of amyloid. Sci Transl Med. 2020; 12(529): eaay 1809 .

15. Davis AA, Inman $C E$, Wargel $Z M$, et al. APOE genotype regulates pathology and disease progression in synucleinopathy. Sci Transl Med. 2020; 12(529): eaay3069.

16. James LM, Georgopoulos AP. The human leukocyte antigen (HLA) DRB1*13:02 allele protects against dementia in continental Western Europe. J Neurol Neuromed. 2019; 4(5): 1-6.

17. James LM, Georgopoulos AP. Tri-Allelic Human Leukocyte Antigen (HLA) Protection Against Dementia. J Neurol Neuromed. 2020; 5(1): 12-17.

18. James LM, Georgopoulos AP. Human leukocyte antigen as a key factor in preventing dementia and associated apolipoprotein E4 risk. Front Aging Neurosci. 2019; 11: e82.

19. James LM, Georgopoulos AP. Persistent antigens hypothesis The human leukocyte antigen (HLA) connection. J Neurol Neuromed. 2018; 3(6): 27-31.

20. James LM, Georgopoulos AP. Dementias caused by persistent pathogens and the role of HLA protection against them. J Neurol Neuromed. 2020; 5(1): 1-11.

21. Gonzalez-Galarza FF, McCabe A, Santos EJ, et al. Allele frequency net database (AFND) 2020 update: gold-standard data classification, open access genotype data and new query tools. Nucleic Acid Research. 2020; 48: D783-8.

22. Charonis S, James LM, Georgopoulos AP. In silico assessment of binding affinities of three dementia-protective Human Leukocyte Antigen (HLA) alleles to nine human herpes virus antigens. Curr Res Transl Med. 2020; doi.org/10.1016/j.retram.2020.06.002

23. Population Reference Bureau. 2016 world population data sheet with a special focus on human needs and sustainable resources. Population Reference Bureau, Washington, DC, 2016. https://www.prb.org/2016world-population-data-sheet/. Accessed February 5, 2019.

24. Snedecor GW, Cochran WG. Statistical Methods. Ames, IA: lowa State Univ. Press; 1989.

25. Paternoster R, Brame R, Mazerolle $P$, et al. Using the correct statistical test for the equality of regression coefficients. Criminolgy. 1998; 36: 859866.

26. Itzhaki RF. Corroboration of a major role for herpes simplex virus type 1 in Alzheimer's disease. Front Aging Neurosci. 2018; 10: e00324. doi: 10.3389/fnagi.2018.00324

27. Eimer WA, Kumar DK, Shanmugam NK, et al. Alzheimer's diseaseassociated $\beta$-amyloid is rapidly seeded by herpesviridae to protect against brain infection. Neuron. 2018; 99(1): 56-63.

28. Bu XL, Wang $X$, Xiang $Y$, et al. The association between infectious burden and Parkinson's disease: A case-control study. Parkinsonism Relat Dis. 2015 ; 21 : 877-881. 
29. Hemling N, Roytta M, Rinne J, et al. Herpesviruses in brains in Alzheimer's and Parkinson's diseases. Ann Neurol. 2003; 54: 267-271.

30. Lindestam Arlehamn CS, Garretti F, Sulzer D, et al. Roles for the adaptive immune system in Parkinson's and Alzheimer's diseases. Curr Opin Immunol. 2019; 59: 115-120. doi: 10.1016/j.coi.2019.07.004.

31. Noble JA, Valdes AM. Genetics of the HLA region in the prediction of type 1 diabetes. Current Diabetes Rep. 2011; 11(6): 533.
32. Balandraud N, Picard C, Reviron D, et al. HLA-DRB1 genotypes and the risk of developing Anti citrullinated protein antibody (ACPA) positive rheumatoid arthritis. PLoS ONE. 2013; 8(5): e64108.

33. Hollenbach JA, Oksenberg JR. The immunogenetics of multiple sclerosis: A comprehensive review. J Autoimmun. 2015; 64: 13-25. 